\title{
Correlation between Diabetic Macular Edema and Best Corrected Visual Acuity in Different Categories of Diabetic Retinopathy
}

\author{
AHMED H.S. ASSAF, M.D.; AHMED A.A. EBEID, M.D.; MOUAMEN M.M. SELEET, M.D. and \\ ELSAYED A.A. MOHAMED, M.Sc. \\ The Department of Ophthalmology, Faculty of Medicine, Ain Shams University
}

\begin{abstract}
Background: Diabetic Retinopathy (DR) is a major cause of vision loss in the working age population and people with diabetes are 25 times more likely than the general population to go blind.
\end{abstract}

Aim of Study: To correlate the Best-Corrected Visual Acuity (BCVA) by ETDRS protocol with macular thickness measurement and morphology as obtained by OCT in a series of patients with diabetic macular edema, with or without diabetic retinopathy.

Patients and Methods: Cross sectional design, in total 102 of 51 selected Egyptian diabetic subjects, 20 males and 31 females were selected by convenient sample in the study during the period from Mars. 2018 to December 2018, all procedures were done at National Institute Of Diabetes And Endocrinology. Eyes enrolled in the study were divided into three groups as follows: Group 1: Eyes with DME and non clinically detectable DR, Group 2: Eyes with DME and NPDR, Group 3: Eyes with DME and PDR.

Results: In the present study,we found that the Diffuse Spongy Macular Edema (DSME) was the most common type of DME, we found a highly significant negative correlation between the CMT and BCVA. There was a significant correlation between the OCT pattern of DME and the severity of diabetic retinopathy. We also found a statistically significant decrease in BCVA in eyes with PDR in comparison to eyes with NPDR and eyes with no clinically detectable diabetic retinopathy.

Conclusion: Based on the data in our study, diffuse spongy macular edema was the most common type of DME with the least CMT and the highest BCVA, we also found the worst BCVA in PDR.

Key Words: Diabetic macular edema-Best corrected visual acuity-Diabetic retinopathy.

\section{Introduction}

DIABETIC Retinopathy (DR) is a major cause of vision loss in the working age population and

Correspondence to: Dr. Elsayed A.A. Mohamed, E-Mail: elsayed.rezq91@gmail.com people with diabetes are 25 times more likely than the general population to go blind [1].

Diabetic Macular Edema (DME) is a major cause of visual acuity loss in diabetes, it affects central vision from the early stages of retinopathy, and it is the most frequent sight-threatening complication of diabetic retinopathy, particularly in older type 2 diabetic patients [2].

Diabetic Macular Edema (DME) is manifested as retinal thickening caused by the accumulation of intraretinal fluid, primarily in the inner and outer plexiform layers. It is believed to be a result of hyper permeability of the retinal vasculature.

The Early Treatment Diabetic Retinopathy Study Group (ETDRS) defined DME (based on clinical grounds) as an increase in retinal thickness at or within one disc diameter of the foveal center whether focal or diffuse, with or without hard exudates, sometimes associated with cysts [3,4].

DME leads to distortion of visual images and may cause a significant decrease in visual acuity even in the absence of severe retinopathy, although macular edema is a common and characteristic complication of diabetic retinopathy and shows apparent association with the systemic metabolic alterations of diabetes, it does not necessarily fit the regular course of diabetic retinopathy progression. It may occur at any stage of diabetic retinopathy [5]

Visual acuity is defined as the "spatial resolving capacity" of the eye or, put another way, the size of an object that can be resolved with an eye. It could be measured by identifying the angle subtended at the eye by the smallest recognizable optotype. 


\section{Visual acuity measurement:}

The most common approach is based on the detailed and rigorous refraction and VA protocols of the Early Treatment Diabetic Retinopathy Study (ETDRS), widely considered the gold standard for assessing VA in the ophthalmic clinical research setting [6].

OCT is a digital optical instrument that generates cross sectional images (tomograms) of the retina by optical-coherence interferometry, a procedure analogous to ultrasound, except for using light (abroad bandwidth near infrared light beam at $840 \mathrm{~nm}$ ) rather than sound, and measures the echo delay time of light reflected and backscattered from the retina. It produces reliable, reproducible, and objective cross sectional images of the retinal structures and the vitreoretinal interface and allows quantitative measurements of Retinal Thickness (RT) $[7,8]$.

\section{OCT classification of DME:}

Type 1 : Diffuse macular edema without cysts.

Type 2: Cystoid macular edema.

Type 3: Tractional macular edema.

Type 4: Serous retinal detachments.

OCT allows a quantitative diagnosis of DME, as it is used to obtain numerical representation of the retinal thickness. CSME may be diagnosed using only biomicroscopy, but CSME with minimal increase in retinal thickness is difficult to recognize without OCT. Different studies demonstrated that OCT may identify DME in patients with normal biomicroscopy [9-11].

In diabetic patients with increased retinal thickness between 200 and $300 \mathrm{~m}$, nonsidering abnormal values if they are above $200 \mathrm{p}, \mathrm{m}$, only $14 \%$ are detected by ophthalmoscopy. It corresponds to a subclinical form of macular edema [12].

\section{Aim of the study:}

To correlate the Best-Corrected Visual Acuity (BCVA) by ETDRS protocol with macular thickness measurement and morphology as obtained by OCT in a series of patients with diabetic macular edema, with or without diabetic retinopathy.

\section{Patients and Methods}

Study design:

Cross sectional design.

\section{Subjects:}

In total 102 of 51 selected Egyptian diabetic subjects, 20 males and 31 females were selected by convenient sample in the study during the period from Mars 2018 to December 2018, all procedures were done at National Institute of Diabetes and Endocrinology. Eyes enrolled in the study were divided into three groups as follows:

- Group 1 : Eyes with DME and non clinically detectable DR.

- Group 2: Eyes with DME and NPDR.

- Group 3: Eyes with DME and PDR.

\section{Inclusion criteria:}

Patients diagnosed with type 1 or 2 diabetes mellitus.

Patients diagnosed with DME.

\section{Exclusion criteria:}

Media opacity like corneal opacity or cataract or vitreous hemorrhage.

Patients with Uveitis, Choroidal Neovascularization $(\mathrm{CNV})$ or glaucoma.

Patient with any ocular or systemic condition predisposing to macular edema.

All participants underwent the following procedures:

History taking (type of DM, on insulin or not, duration, previous laser, previous intravitreal injection, smoking, hypertensive or not).

Refraction and best correction visual acuity measurement by ETDRS chart.

Slit lamp examination.

Intraocular pressure by Goldman applanation tonometer.

Fundus examination to diagnose CSME and classify diabetic retinopathy.

Blood pressure measurement, $\mathrm{CBC}$, renal function test.

Changes in retinal thickness and morphology assessed by Optical Coherence Tomography (OCT NIDEK RS 3000 advance).

Statistical analysis of the present study was conducted using the mean, standard deviation, student $t$-test, Chi-square and linear correlation coefficient tests by SPSS software package version. 


\section{Results}

Table (1): Comparison between DR grades as regards type of edema.

\begin{tabular}{llllllllll}
\hline & \multicolumn{2}{c}{ No } & \multicolumn{2}{c}{ NPDR } & \multicolumn{2}{c}{ PDR } & \multicolumn{2}{c}{ Chi square test } \\
\cline { 2 - 9 } & No & $\%$ & No & $\%$ & No & $\%$ & $x^{2}$ & $p$-value \\
\hline CME & 7 & 23.3 & 10 & 26.3 & 14 & 41.2 & 2.874 & 0.238 \\
NSD & 0 & 0.0 & 1 & 2.6 & 7 & 20.6 & 11.622 & 0.003 \\
Diffuse & 24 & 80.0 & 30 & 78.9 & 21 & 61.8 & 3.636 & 0.162 \\
Tractional & 0 & 0.0 & 0 & 0.0 & 3 & 8.8 & 6.182 & 0.045 \\
& 0.146 & 0.228 & 0.001 & & \\
\hline
\end{tabular}

Of the 38 eyes with NPDR, 10 eyes $(26.3 \%)$ had CME, one eye (2.6\%) had NSD, and 30 eyes (78.9) had diffuse macular edema while non of them had tractional macular edema.

Table (2): Correlation between types of macular edema with CMT \& BCVA.

\begin{tabular}{lccccccc}
\hline & \multicolumn{2}{c}{$\begin{array}{c}\text { Central } \\
\text { Macular } \\
\text { thickness }\end{array}$} & BCVA & \multicolumn{2}{c}{$\begin{array}{c}\text { Independent } \\
t \text {-test }\end{array}$} \\
\cline { 2 - 8 } & Mean & SD & Mean & SD & $t$ & $p$-value \\
\hline CME & 420.41 & 134.40 & 0.54 & 0.21 & -17.394 & 0.001 \\
NSD & 502.25 & 142.72 & 0.57 & 0.19 & -9.942 & 0.001 \\
Diffuse & 300.27 & 106.24 & 0.27 & 0.21 & -24.455 & 0.001 \\
Tractional & 454.67 & 112.07 & 0.62 & 0.10 & -7.017 & 0.002 \\
\hline
\end{tabular}

There was clinically significant correlation between types of edema and CMT \& BCVA.

Table (3): Relation between BCVA as regards type of edema.

\begin{tabular}{lcccc}
\hline & \multicolumn{2}{c}{ BCVA } & \multicolumn{2}{c}{ Independent $t$-test } \\
\cline { 2 - 5 } & Mean & SD & $t$ & $p$-value \\
\hline CME & 0.54 & 0.21 & -9.162 & 0.001 \\
NSD & 0.57 & 0.19 & -3.684 & 0.001 \\
Diffuse & 0.27 & 0.21 & 3.135 & 0.002 \\
Tractional & 0.62 & 0.10 & -2.490 & 0.015 \\
\hline
\end{tabular}

As regards the BCVA, there was a statistically significant decrease in BCVA in CME, tractional and NSD in comparison to diffuse macular edema with the worst VA in tractional macular edema.

Table (4): Correlation between DR grades and BCVA.

\begin{tabular}{lcclcc}
\hline & \multicolumn{2}{c}{ BCVA } & & \multicolumn{2}{c}{ One way ANOVA } \\
\cline { 2 - 3 } \cline { 6 - 6 } & Mean & SD & & $\mathrm{f}$ & $p$-value \\
\hline No & 0.21 & 0.14 & & 5.791 & 0.004 \\
NPDR & 0.30 & 0.23 & & \\
PDR & 0.40 & 0.25 & & \\
\hline
\end{tabular}

Regarding the grade of DR there was a statistically significant decrease in BCVA in eyes with PDR in comparison to eyes with NPDR and eyes with no clinically detectable diabetic retinopathy.
Table (5): Correlation between CMT and BCVA.

\begin{tabular}{ccc}
\hline & \multicolumn{2}{c}{ BCVA } \\
\cline { 2 - 3 } & \multicolumn{2}{c}{ Value } \\
\hline Central macular thickness & 0.726 & 0.001 \\
\hline
\end{tabular}

A highly statistically significant correlation was found between the CMT \& BCVA.

Table (6): Relation between CMT and type of edema.

\begin{tabular}{lcccc}
\hline & \multicolumn{4}{c}{ Central macular thickness Independent $t$-test } \\
\cline { 2 - 5 } & Mean & SD & $t$ & $p$-value \\
\hline CME & 420.41 & 134.40 & -7.951 & 0.001 \\
NSD & 502.25 & 142.72 & -5.721 & 0.001 \\
Diffuse & 300.27 & 106.24 & 2.578 & 0.012 \\
Tractional & 454.67 & 112.07 & -2.232 & 0.028 \\
\hline
\end{tabular}

Regarding the CMT and type of edema there was a statically significant increase in CMT in eyes with NSD, tractional and cystiod in comparison to eyes with diffuse macular edema.

Table (7): Relation between age as regards type of edema.

\begin{tabular}{llllc}
\hline & \multicolumn{2}{c}{ Age } & \multicolumn{2}{c}{ Independent $t$-test } \\
\cline { 2 - 5 } & Mean & SD & \multicolumn{1}{c}{$t$} & $p$-value \\
\hline CME & 47.34 & 8.61 & 0.220 & 0.826 \\
NSD & 41.13 & 13.23 & 2.253 & 0.026 \\
Diffuse & 47.92 & 8.26 & -0.511 & 0.610 \\
Tractional & 31.67 & 10.69 & 3.393 & 0.001 \\
\hline
\end{tabular}

There was no statistically significant correlation between types of edema and age.

Table (8): Correlation between previous IVI and types of edema.

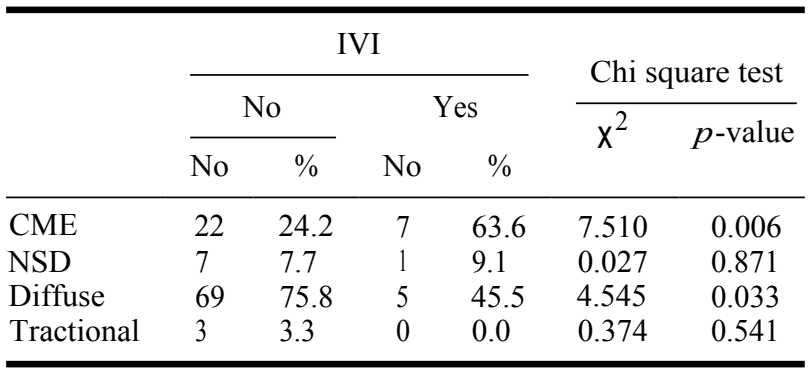

Of 11 eyes that had received previous IVI, 7 eyes $(63.6 \%)$ have CME, 5 eyes $(45.5 \%)$ have diffuse macular edema and 1 eye have NSD.

\section{Discussion}

In this study, we compared the BCVA in different morphological types of DME classified by OCT in diabetic patients, whether or not they had clinically detectable diabetic retinopathy. 
The study was conducted on 102 eyes of 51 patients with CSME, 20 of which were males and 31 were female with a mean age of 47 years. They were divided into three groups, PDR group, NPDR group and non clinically detectable DR group. All procedures were done at National Institute of Diabetes and Endocrinology.

In the current study Diffuse Spongy Macular Edema (DSME) was found in (73.5\%) of the eyes whereas CME was found in $30.4 \%$ of the eyes, SRD in $7.0 \%$, and tractional Macular edema in $3.2 \%$ of eyes.

DSME was also the most common type in previous reports that studied the OCT patterns of DME. In their study, Otani et al. [13] found DSME in $88 \%$ of the eyes, CME in $47 \%$, and SRD in $15 \%$, while Yamamoto et al. [14] in their study reported DSME in $60 \%$ and CME in $40 \%$ of the eyes with DME. In addition, the study by Murakami et al. [15] also classified three types of morphology at the presumed fovea: CME (CME type: 16\%), SRD (SRD type: $16.8 \%$ ), and the absence of either CME or SRD (diffuse type: $67.2 \%$ ).

It has been established that central macular thickening can be associated with a decrease in VA [16]

In this study, we found a highly significant negative correlation between the CMT and BCVA.

We also found that patients with tractional macular edema had the worst BCVA followed by patients with SRD then CME. The highest BCVA was found in the DSME group.

When we studied our findings regarding the BCVA and type of edema with the CMT we found that they correlated well, with the worst BCVA \& CMT seen in CME and SRD and the best BCVA \& CMT seen in DSME.

The only exception was in patients with tractional macular edema, as they had more severe drop of vision while not having the highest CMT. This severe drop of vision may be explained by the macular \& foveal distortion caused by the fibro vascular traction and not just the CMT.

These result was comparable to previous studies, Yamamoto et al. [14] found that the fovea of the eyes with cystoid edema was significantly thicker than the fovea of eyes with diffuse swelling.

In the study by Kim et al. [17], the mean retinal thickness and mean visual acuities also varied between groups, depending on the morphologic pattern, increasing retinal thickness in all patterns was significantly correlated with worse VA.

Otani et al. [13], also reported that the retinal thickness at the central fovea and the BCVA showed an intermediate negative correlation regardless of the different morphological features.

Whereas Murakami et al. [15] concluded that the mean log MAR VA with the CME type was significantly worse than with the SRD type or diffuse type but found that parafoveal thickening was significantly correlated with poor VA in CME type and diffuse type but not in the SRD type.

In the present study, there was a significant correlation between the OCT pattern of DME and the severity of diabetic retinopathy; we found a higher number of eyes with SRD \& tractional macular edema in the PDR group than NPDR \& non clinically detectable DR groups. However, there was no statistically difference between the three groups concerning the number of eyes with CME \& DSME.

This can be explained by the retinal ischemia in eyes with PDR release endogenous vascular permeability factors that break down the bloodretinal barrier and cause severe leakage from damaged capillaries. In addition, the deterioration of retinal pigment epithelium function from ischemia may play a role in serous retinal detachment as well as the rapidity of the increase in intraretinal fluid.

This may explain the finding of Catier et al. [18] that in macular edema associated with central retinal vein occlusion, serous retinal detachment was more frequent and higher than in diabetic macular edema. Moreover, subretinal fluid could be of tractional origin instead of serous, but in our study, tractional macular edema was present in only $3.2 \%$ of cases.

In comparison to study of Alkuraya et al. [19] found significant association between OCT pattern of clinically significant diabetic macular edema and severity of concurrent retinopathy. However, Kang et al. [20] did not show a significant correlation between the distribution of OCT types and the stages of diabetic retinopathy.

In this study, we found a statistically significant decrease in BCVA in eyes with PDR in comparison to eyes with NPDR and eyes with no clinically detectable diabetic retinopathy. Compared to study by Bénédicte Dupas et al. [21] patients with severe non proliferative or proliferative diabetic retinop- 
athy, decreased VA may be associated with the degree of capillary loss in the deep capillary complex.

\section{Conclusion:}

Based on the data in our study, we found that the BCVA and CMT correlated well the type of edema, with the worst BCVA \& CMT seen in CME and SRD and the best BCVA \& CMT seen in DSME.

The only exception was in patients with tractional macular edema, as they had more severe drop of vision while not having the highest CMT.

In the present study, there was a significant correlation between the OCT pattern of DME and the severity of diabetic retinopathy; we found a higher number of eyes with SRD \& tractional macular edema in the PDR group than NPDR \& non clinically detectable DR groups. However, there was no statistically difference between the three groups concerning the number of eyes with CME \& DSME.

We also found a statistically significant decrease in BCVA in eyes with PDR in comparison to eyes with NPDR and eyes with no clinically detectable diabetic retinopathy.

\section{References}

1- Diabetes UK. Cost of diabetes. 2014 http://www.diabetes. . co.uk/cost-of-diabetes.html (accessed $27 \mathrm{Feb} 2015$ ).

2- AIELLO L.P., GARDNER T.W., KING G.L., BLANKENSHIP G., CAVALLERANO J.D., FERRIS F.L.III and KLEIN R.: Diabetic retinopathy. Technical review. Diabetes Care, 21: 143-56, 1998.

3- Early Treatment Diabetic Retinopathy Study Research Group: Grading diabetic retinopathy from stereoscopic color fundus photographs-an extension of the modified Airlie House classification. ETDRS report number 10. Ophthalmology, 98 (5 Suppl): 786-806, 1991.

4- Early Treatment Diabetic Retinopathy Study Group: Photocoagulation for diabetic macular edema: Early treatment diabetic retinopathy study report No 1 . Arch. Ophthalmol., 103 (12): 1796-806, 1985.

5- KLEIN B.E., KLEIN R. and LEE K.E.: Components of the metabolic syndrome and risk of cardiovascular disease and diabetes in beaver dam. Diabetes Care, 25 (10): 17904, 2002.

6- FERRIS F.L., KASSOFF A., BRESNICK G.H. and BAILEY I.: New visual acuity charts for clinical research. Am. J. Ophthalmol., 94: 91-6, 1982.

7- YAMAMOTO S., YAMAMOTO T., HAYASHI M. and TAKEUCHI S.: Morphological and functional analyses of diabetic macular edema by optical coherence tomography and multifocal electroretinograms. Graefes Arch. Clin. Exp. Ophthalmol., 239 (2): 96-101, 2001.
8- KIM B.Y., SMITH S.D. and KAISER P.K.: Optical coherence tomographic patterns of diabetic macular edema. Am. J. Ophthalmol., 142 (3): 405-12, 2006.

9- LANG G.E.: Optical coherence tomography findings in diabetic retinopathy, in Diabetic Retinopathy, Vol. 39, ed. by G.E. Lang. Dev Ophthalmol (Karger, Basel), pp. 31-47, 2007.

10- YANG C.S., CHENG C.Y., LEE F.L., HSU W.M. and LIU J.H.: Quantitative assessment of retinal thickness in diabetic patients with and without clinically significant macular edema using optical coherence tomography. Acta Ophthalmol. Scand., 79 (3): 266-70, 2001.

11- SCHAUDIG U.H., GLAEFKE C., SCHOLZ F. and RICHARD G.: Optical coherence tomography for retinal thickness measurement in diabetic patient without clinical significant macular edema. Ophthalmic Surg. Lasers., 31 (3): 182-6, 2000.

12- BROWN J.C., SOLOMON S.D., BRESSLER S.B., SCHACHAT A.P., DiBERNARDO C. and BRESSLER N.: Detection of diabetic foveal edema, contact lens biomicroscopy compared with optical coherence tomography. Arch. Ophthalmol., 122 (3): 330-5, 2004.

13- OTANI T., KISHI S. and MARUYAMA Y.: Patterns of diabetic macular edema with optical coherence tomography. Am. J. Ophthalmol., 127: 688-93, 1999.

14- YAMAMOTO S., YAMAMOTO T., HAYASHI M. and TAKEUCHI S.: Morphological and functional analyses of diabetic macular edema by optical coherence tomography and multifocal electroretinograms. Graefes Arch. Clin. Exp. Ophthalmol., 239: 96-101, 2001.

15- MURAKAMI T., NISHIJIMA K., SAKAMOTO A., OTA M., HORII T. and YOSHIMURA N.: Association of pathomorphology, photoreceptor status, and retinal thickness with visual acuity in diabetic retinopathy. Am. J. Ophthalmol., 151: 310-7, 2011.

16- BROWNING D.J., GLASSMAN A.R., AIELLO L.P., BECK R.W., BROWN D.M., FONG D.S., et al.: Diabetic Retinopathy Clinical Research Network Relationship between optical coherence tomography-measured central retinal thickness and visual acuity in diabetic macular edema. Ophthalmology, 114: 525-36, 2007.

17- KIM B.Y., SMITH S.D. and KAISER P.K.: Optical coherence tomographic patterns of diabetic macular edema. Am. J. Ophthalmol., 142: 405-12, 2006.

18- CATIER A., TADAYONI R., PAQUES M., ERGINAY A., HAOUCHINE B., GAUDRIC A., et al.: Characterization of macular edema from various etiologies by optical coherence tomography. Am. J. Ophthalmol., 140: 200-6, 2005.

19- ALKURAYA H., KANGAVE D. and EL-ASRAR A.M. The correlation between optical coherence tomographic features and severity of retinopathy, macular thickness and visual acuity in diabetic macular edema. International ophthalmology, 26 (3): 93-9, 2005.

20- KANG S.W., PARK C.Y. and HAM D.I.: The correlation between fluoresce angiographic and optical coherence tomographic features in clinically significant diabetic macular edema. Am. J. Ophthalmol., 137: 313-22, 2004. 
21- DUPAS B., MINVIELLE W., BONNIN S., COUTURIER A., ERGINAY A., MASSIN P., GAUDRIC A. and

TADAYONI R.: Association between vessel density and visual acuity in patients with diabetic retinopathy and poorly controlled type 1 diabetes. JAMA Ophthalmology, 136 (7): 721-8, 2018.

\section{الإرتباط بين إرتشاح مقولة العين السكرى وآفضل تصحيح لحدة الإبصار

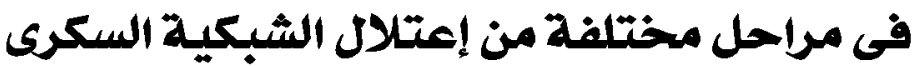

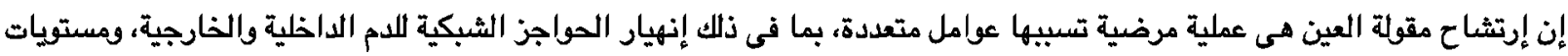

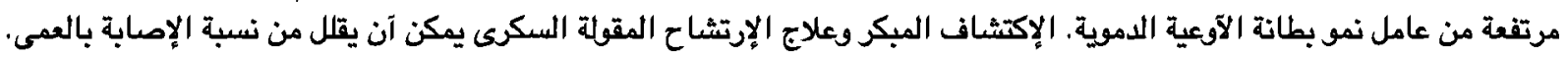

التصوير المقطعى التماسك البصرى يقوم بقياس وقت تآخر الصدى المنعكس والمبعثر من الشبكية، وينتج صوراً مقطعية موثوقة وقابلة اللتكرار وموضوعية للهياكل الشبكية والسطح البينى بين الشبكية الجسم والجسم الزجاجى ويسمع بإجراء قياسات كمية لسمك الشبكية.

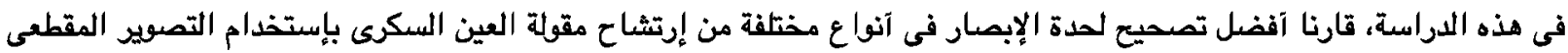
التماسك البصرى فى حالة وجود إعتلال الشبكية لسكرى آو لا.

وقد آجريت الدراسة على ج ـا عين فى اه من المصريين المصابين بمرض السكرى ويعانوا من إرتشاح سكرى بمقولة العين، .ب وتم تقسيم الدراسة إلى: • إرتشاح مقولة العين السكرى مع عدم وجود إعتلال شبكية سكرى بالفحص السريرى. • • إرتشاح مقولة العين السكرى مع وجود إعتلال شبكية سكرى غير العير تكاثرى.

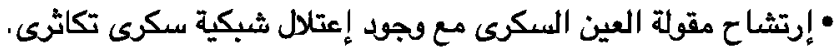

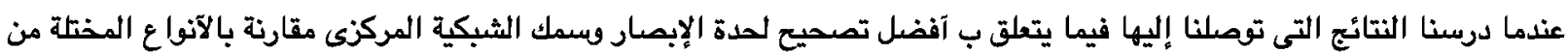

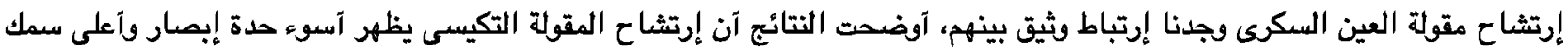

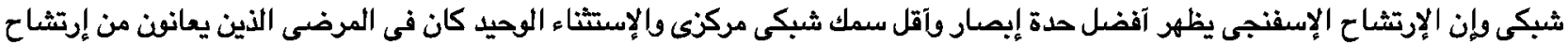

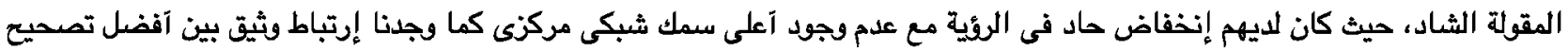

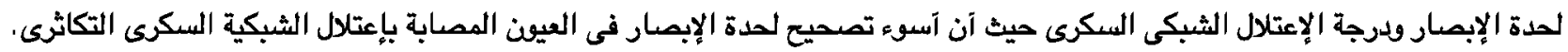

